\title{
MicroRNAs: promising biomarkers for diagnosis and therapeutic targets in human colorectal cancer metastasis
}

\author{
Keun Hur* \\ Department of Biochemistry and Cell Biology, Cell and Matrix Research Institute, School of Medicine, Kyungpook National University, \\ Daegu 700-842, Korea
}

\begin{abstract}
Colorectal cancer (CRC) is the third most common cancer and the fourth most common cause of cancer-related death worldwide. Distant metastasis is a major cause of mortality in CRC. MicroRNAs (miRNAs) are small non-coding RNA molecules involved in the post-transcriptional and translational regulation of gene expression. Many miRNAs are aberrantly expressed in cancer and influence tumor progression. Accumulating studies suggest that multiple miRNAs are actively involved in the CRC metastasis process. Thus, we aim to introduce the role of miRNAs in multi-steps of CRC metastasis, including cancer cell invasion, intravasation, circulation, extravasation, colonization, angiogenesis, and epithelial-mesenchymal transition (EMT). Moreover, we suggest the potential application of miRNAs as biomarkers for CRC patients with metastasis. [BMB Reports 2015; 48(4): 217-222]
\end{abstract}

\section{INTRODUCTION}

MicroRNAs (miRNAs) are small (20-22 nucleotides) non-coding single-strand RNAs involved in the post-transcriptional and translational regulation of gene expression. MiRNA is transcribed by RNA polymerase II in the nucleus into primary miRNA (pri-miRNA) containing a 7-methylguanosine cap and a $3^{\prime}$ poly(A) tail end. In the nucleus, pri-miRNA is further processed by the endonuclease Drosha, together with its double-stranded RNA (dsRNA)-binding protein partner DGCR8 (in mammals) to produce precursor miRNA (pre-miRNA) $(1,2)$. The pre-miRNA is then transported into the cytoplasm by the transporting protein exportin-5. In the cytoplasm, the pre-miRNA is further processed by another endonuclease Dicer together with its dsRNA-binding partner TRBP (transactivation-response RNA-binding protein; in mammals) into mature double-stranded miRNA. The double-stranded miRNA (miRNA-miRNA* com-

*Corresponding author. Tel: +82-53-420-4821; Fax: +82-53-4221466; E-mail: KeunHur@knu.ac.kr

http://dx.doi.org/10.5483/BMBRep.2015.48.4.007

Received 13 January 2015

Keywords: Biomarker, Colorectal cancer, Metastasis, MicroRNA plex) is loaded into an Argonaute (AGO) protein. Finally, the mature single strand miRNA (also called the guide strand) incorporated into the RNA-induced silencing complex (RISC), whereas the other passenger strand (denoted with an asterisk at the end of the name) is degraded. This miRNA/RISC complex is responsible for miRNA function. MiRNAs negatively regulate target genes by binding to complementary sequences in the 3'-untranslated region (3'-UTR) of the target mRNA. Approximately, 700 miRNAs have been identified in humans, and more than $30 \%$ of protein-coding genes are regulated by miRNA (3).

Colorectal cancer (CRC) is the third most common cancer and the fourth most common cause of cancer-related death worldwide (4). Although earlier diagnosis and improved treatment regimens have increased survival rates in patients with CRC, approximately $50 \%$ of patients who are diagnosed with CRC will experience local or systemic recurrence due to metastatic disease (5). Distant metastasis is a major cause of death in CRC patients, and the liver is the most common metastatic site for patients with CRC. Thus, aggressive surgical resection of hepatic metastases improves 5-year overall survival rates, which range from $25 \%$ to $40 \%$ (6). Nonetheless, about onethird of CRC patients with liver metastases still experience tumor recurrence.

In recent years, multiple studies have been demonstrated the key role of miRNAs in various physiological and pathological processes in CRC. Several miRNAs are directly involved in the regulation of the primary pathways of CRC development. Inactivation of adenomatous polyposis coli (APC) has been found in more than $60 \%$ of CRC patients, which is associated with upregulation of miR-135a/b in colonic epithelial cells (7). Loss of tumor protein 53 (p53) gene is observed during progression of adenoma to adenocarcinoma, and it mediates the function of p53 in apoptosis and cell cycle arrest via a direct downstream target, miR-34a (8). Additionally, upregulation of c-myc enhances adenoma to adenocarcinoma transition, which is regulated by the miR-17-92 cluster (miR-17, miR-18a, miR-19a/b, miR-20a, and miR-92a) (9). Augmentation and inactivation of the phosphatidylinositol 3-kinase (PI3K) pathway have been linked with miR-21 and miR-126, respectively $(10,11)$. KRAS knockdown and EGFR-MAPK pathway activation are associated with let-7, miR-18a*, and miR-

ISSN: 1976-670X (electronic edition)

Copyright (C) 2015 by the The Korean Society for Biochemistry and Molecular Biology

(c) This is an open-access article distributed under the terms of the Creative Commons Attribution Non-Commercial License (http://creativecommons.org/licenses/by-nc/3.0) which permits unrestricted non-commercial use, distribution, and reproduction in any medium, provided the original work is properly cited. 
143 (12-14). Moreover, much accumulating evidence suggests that miRNAs are not only involved in colorectal tumorigenesis, but also multiple miRNAs actively modulate CRC metastasis. Thus, in this article, we will review the emerging role of miRNAs in the metastatic process of CRC as well as the potential application of miRNAs as biomarkers for the diagnosis and therapeutics of CRC patients with metastasis.

\section{MiRNAs IN A MULTI-STEP PROCESS OF CRC METASTASIS}

Cancer metastasis is a multi-step process that involves (i) local invasion of cancer cells, (ii) migration of cancer cells into blood vessels, (iii) survival in the circulatory system, (iv) extravasation, and (v) colonization of distant organs (15). Notably, each of these events involves a number of dysregulation of miRNAs. Here, we will describe in detail the functional role of miRNAs in each metastasis step (Table 1).

Local invasion of cancer cells into adjacent tissues is the first step in the metastasis cascade. During this process, activation of several proteolytic enzymes, including extracellular matrix (ECM)-degrading proteases, is required (16). Various miRNAs that control the expression of these enzymes have been indentified. Overexpression of miR-21 is commonly observed in human CRC, which is correlated with CRC metastasis development. In CRC cell lines, high miR-21 expression positively regulates invasion and migration ability. Mir-21 directly targets several tumor suppressor genes including programmed cell death 4 (PDCD4) $(17,18)$, sprouty homolog 2 (SPRY2) (19), tropomyosin 1 (TPM1) (20), chemokine (C-C motif) ligand 20 (CCL2) (21), cell division cycle 25A (CDC25A) (22), maspin, phosphatase, and tensin homolog (PTEN) (23), and metallopeptidase inhibitor 3 (TIMP3) (24). Higher miR-21 expression enhances CRC cell migration and invasion, whereas suppression of the miRNA attenuates these effects $(17,25,26)$. Additionally, upregulation of miR-31 is known to positively regulate invasion and migration properties of CRC cells in vitro as well as in vivo. The T lymphoma invasion and metastasis 1 (TIAM1) gene has been suggested to be a target of both miR-21 and miR-31 (27). In CRC cells, hypoxia induced both miR-103 and miR-107 expression that directly target death-associated pro- tein kinase (DAPK) and krüppel-like factor 4 (KLF4) genes resulted in enhancing cell to matrix adhesion and cell motility (28). Moreover, miR-29a also targets the KLF4 gene, and it was upregulated in CRC tissues $(29,30)$. Increased expression of miR-122 was reported from non-neoplastic tissue to dysplasia, but downregulated from dysplasia to CRC, indicating that miR-122 expression is possibly involved in spreading CRC cells to adjacent tissues (31).

Once cancer cells successfully pass through the basement membrane and the extracellular matrix surrounding the tumor, they reach the blood vessels (or lymphatics). Subsequently, cancer cells invade and move through the blood vessels, which is termed intravasation. The process of intravasation is less well investigated. Only one miRNA, miR-21 seems to be involved in this process. As described above, miR-21 suppresses the PDCD4 tumor suppressor gene (17). Elevated miR-21 expression was associated with higher potential of CRC metastasis as well as lymph node metastasis in CRC patients $(25,32)$.

During blood circulation, cancer cells are exposed to the mechanical stresses of blood flow and the immune system. Some cancer cells exit the blood circulation and invade the blood vessel again at a distant location, which is known as extravasation (the reverse of the intravasation process). Then, the cancer cells finally develop a secondary tumor at the distant organ. Although the function of miRNAs in survival of cancer cells in blood circulation and the extravasation process has not been investigated, few miRNAs are considered to be key factors in the process. Expression of miR-126 has been reported in human umbilical vein endothelial cells (HUVECs) (33). MiR-126 directly targets the vascular cell adhesion molecule 1 (VCAM-1) gene that mediates the adhesion of lymphocytes to vascular endothelium, which may be helpful to circulating cancer cells (33). Other miRNAs are miR-155 and the miR-17-92 cluster (34). Those miRNAs play a key role in the differentiation of $B$ cells as well as the regulation of $T$ cell lineage induction pathways, which may support immune evasion of cancer cells in blood circulation.

The final step of cancer metastasis is colonization of the extravasated cancer cells at secondary sites. Eventually, cancer cells have to survive and form tumors at the new location,

Table 1. MicroRNAs involved in CRC metastasis

\begin{tabular}{|c|c|c|c|c|c|c|c|}
\hline \multicolumn{2}{|c|}{ Local invasion } & \multicolumn{2}{|c|}{ Intravasation, Circulation, Extravasation } & \multicolumn{2}{|c|}{ Colonization } & \multicolumn{2}{|c|}{ Angiogenesis } \\
\hline microRNAs & Reference & microRNAs & Reference & microRNAs & Reference & microRNAs & Reference \\
\hline miR-21 & $(17,25,26)$ & miR-17-92 cluster & (34) & miR-34a & (35) & miR-17-92 cluster & (46) \\
\hline miR-31 & (27) & miR-21 & $(17,25,32)$ & miR-146a & (36) & miR-107 & $(42,43)$ \\
\hline miR-103 & (28) & miR-126 & (33) & miR-449b & (38) & miR-145 & $(42,43)$ \\
\hline miR-107 & (28) & miR-155 & (34) & miR-451 & (37) & miR-194 & (45) \\
\hline miR-122 & (31) & & & & & $\operatorname{miR}-497$ & (44) \\
\hline
\end{tabular}


known as micrometastases. During this process, cancer cells need to activate various cell signaling pathways to enter the cell cycle and to achieve high cell proliferation. In this regard, the presence of cancer stem cell populations has been issued in cancer metastasis. Cancer stem cells are characterized by their ability to differentiate and self-renew. Recently, an increasing number of studies has reported the existence of CRC stem cells, and miRNAs are known to regulate the 'stemness' of cancer cells. MiR-34a acts as a cell-fate determinant to choose between self-renewal and differentiation in early-stage CRC stem cells (35). More recently, it has been demonstrated that miR-146a modulates the symmetrical division of CRC stem cells and attenuates tumorigenicity (36). Additionally, miR-451 was downregulated in CRC stem cells, and it was associated with self-renewal ability, tumorigenicity, and chemoresistance to irinotecan (37). Moreover, miR-449b suppressed the proliferation of SW1116 CRC stem cells via downregulation of CCND1 and E2F3 expression (38). Thus, diverse miRNAs may be deeply involved in metastasized cancer cell survival in distant organs.

\section{MiRNAs IN THE REGULATION OF ANGIOGENIC FACTORS}

Once cancer cells reach distant organs, they induce the growth of new blood vessels to obtain a new blood supply. A blood supply is needed to obtain the oxygen and nutrients necessary for tumor growth. Angiogenesis is the formation of new blood vessels, which is activated by both pro-angiogenic factors, including vascular endothelial growth factor (VEGF), basic fibroblast growth factor (bFGF), angiogenin, transforming growth factor (TGF)- $\alpha$, TGF- $\beta$, tumor necrosis factor (TNF)- $\alpha$, and insulin-like growth factor 1 receptor (IGF1-R), and it can be inhibited by anti-angiogenic factors, such as angiostatin, endostatin, interferon, platelet factor 4, and thorombospondin-1 (TSP-1) (39-41).

Several studies have indicated that miRNAs modulate the angiogenesis process by targeting diverse crucial angiogenic factors. Hypoxia induces neovascularization in tumor cells, which stimulates expression of pro-angiogenic molecules, such as VEGF. Hypoxia inducible factor-1 (HIF-1) plays a key role in this process, and miR-107 and miR-145 were found to negatively regulate HIF-1 in CRC $(42,43)$. Another pro-angiogenic factor is IGF1-R, which is targeted by miR-497 (44). Down-regulation of IGF1-R has been reported in CRC cells versus normal colonic mucosa, which increased cell apoptosis and suppressed cell proliferation and invasion ability (44). On the other hand, thorombospondin (THBS1), which encodes TSP-1, acts as an anti-angiogenic factor through regulation of microvascular density and vessel size. Up-regulation of miR-194 by p53 negatively regulates THBS1 (45). Additionally, the miR-17-92 cluster is frequently amplified in multiple cancers, including CRC, which enhances angiogenesis via directly targeting TSP-1 (46).

\section{MiRNAs AS REGULATORS OF THE EPITHELIAL-MESENCHYMAL TRANSITION (EMT) PROCESS IN CRC}

The epithelial-mesenchymal transition (EMT) is a process of converting immotile epithelial cancer cells to motile mesenchymal cancer cells. EMT is characterized by loss of epithelial features including cell adhesion, down-regulation of epithelial markers (E-cadherin, plakoglobin), and acquisition of mesenchymal markers (vimentin, $\mathrm{N}$-cadherin, fibronectin), and increased cell motility and invasiveness (47). Several cell signaling pathways, including Wnt, Notch, TGF- $\beta$, hepatocyte growth factor (HGF), and platelet derived growth factor (PDGF), are involved in EMT initiation (48, 49). Various transcription factors, such as TWIST, SNAI1, SNAI2 (also known as SLUG), ZEB1, and ZEB2, have been reported to be inducers of the EMT process.

Recently, miRNAs have emerged as new EMT regulators. One of the best known EMT-related miRNAs is the miR-200 family (miR-200a, miR-200b, miR-200c, miR-141, and miR-429) (50-52). Expression of these miRNA members is known to lead to an epithelial cellular phenotype in CRC cells. Park et al. demonstrated that suppression of the miR-200 family induced EMT in the HCT116 CRC cell line, by directly targeting ZEB1 (52). More recently, we systematically investigated the role of the miR-200 family in the development of CRC metastasis (53). Hypomethylation of miR-200c promoter region allowed up-regulation of miR-200c expression in liver metastasis tissues, compared with matched corresponding primary CRC tissues. High miR-200c expression suppressed direct target genes (ZEB1, ETS1, and FLT1), which, in turn, regulated E-cadherin and vimentin expression to trigger a MET ('reverse EMT') process in metastasized CRC cells. Interestingly, the ZEB1/2 transcription factors bind to the miR-200 promoter region and regulate their expression, which results in a ZEB/miR-200 double-negative feedback loop (50, 51, 54). Beyond the miR-200 family, Rokavec et al. identified an IL-6R/STAT3/miR-34a feedback loop, and it was required to progress the EMT process in CRC cell lines (55).

\section{CLINICAL APPLICATION OF MiRNAs AS BIOMARKERS}

Currently, measuring plasma carcinoembryonic antigen (CEA) levels and/or the fecal occult blood test (FOBT) are widely used as non-invasive diagnostic tools for CRC patients (56-58). However, these methods are not sufficient, due to a lack of sensitivity as well as specificity for screening an average CRC risk population. High CEA levels could be detectable in response to inflammation, such as inflammatory bowel disease (IBD) and CEA may not be elevated in advanced-stage CRC patients. Additionally, FOBT revealed relatively poor sensitivity at detecting early stage CRC (59). Indeed, randomized clinical trials indicated that FOBT is not reliable and that it only reduces CRC mortality by $30 \%$ (60).

In this context, miRNAs are emerging as ideal disease bio- 
markers for diagnosis as well as therapeutics in CRC patients. MiRNAs are highly stable in various bodily fluids, including serum, plasma, urine, tears, breast milk, and saliva. Moreover, the expression of miRNAs is specific to tissues or tumor stages, and the level of miRNAs can be readily assessed by various simple methods, including real-time reverse transcription polymerase chain reaction (qRT-PCR). Several recent studies have investigated blood circulating miRNAs and have demonstrated their clinical relevance for the diagnosis of CRC patients. Huang et al. identified that high plasma miR-29a and miR-92a expression are useful non-invasive biomarkers to distinguish CRC from healthy controls (30). Additionally, eight elevated plasma miRNAs in patients with colonic polyps were reported by Kanaan et al. (61). Among them, three plasma miRNAs (miR-15b, miR-139-3p, and miR-431) could discriminate Stage IV CRC patients from normal controls. Likewise, Wang et al. identified a panel of plasma miRNAs (miR-409-3p, miR-7, and miR-93) that displayed high diagnostic accuracy in discriminating CRC from a healthy group (62). More recently, Toiyama et al. determined a potential role of serum miR-21 expression as a biomarker for the early detection and prognosis of CRC (63).

Unlike circulating miRNAs for diagnosis and prognosis of CRC, non-invasive miRNAs biomarkers for CRC recurrence and metastasis have not been well investigated. In order to understand the molecular mechanisms of metastasized cancer cells and to discover specific miRNAs originating from the metastasized tumor region, the primary cancer region should be compared directly with the metastasized cancer region from the same individual. However, due to the difficulty of gathering such clinical samples, most of CRC metastasis studies have been conducted based on primary CRC tissues. Previously, we have demonstrated that miR-200c is overexpressed in liver metastasis, compared with matching primary CRC tissue specimens (53). In a further validation study for miR-200c expression in serum samples from CRC patients, serum miR-200c expression was an independent predictor of lymph node metastasis and tumor recurrence (64). More recently, we performed comprehensive discovery for expression patterns of metastasis-specific miRNAs by directly comparing primary CRC and matched liver metastases. Finally, we demonstrated that miR-885-5p is significantly up-regulated in liver metastases compared with primary CRC tissues, and high serum miR-885-5p levels predicted CRC prognosis as well as metastasis (65).

Because miRNAs have been shown to play critical functional roles in CRC carcinogenesis and metastasis, they have been considered as therapeutic targets as well. There are two general strategies for miRNA-based therapeutics: blocking oncogenic miRNAs and restoration of tumor-suppressor miRNAs. For the inhibition of miRNAs, several approaches to directly block specific miRNAs have been developed, including anti-miRNA oligonucleotides (AMOs), miRNA sponges, miRNA masks (target protectors), and small molecule inhibitors of miRNAs. In CRC cell lines, blocking of oncogenic miRNAs (miR- 20a, miR-21, miR-31, miR-95, and miR-675) by anti-miRNAs suppressed cell proliferation, transformation, migration, and metastasis, whereas it enhanced chemosensitivity $(17,66)$. In contrast, down-regulated miRNAs can be restored by introducing synthetic oligonucleotides, such as miRNA precursors and miRNA mimics. Elevation of miR-34a using mimic transfection in the DLD-1 CRC cell line showed inhibition of cell growth, invasion, and migration abilities, but enhanced sensitivity to 5-fluorouracil (67). Likewise, restoration of miR-143 using a miR-143 precursor reduced SW480 CRC cell line proliferation as well as suppressed DLD-1 CRC cell line xenograft tumor growth $(68,69)$. Nonetheless, miRNA-based therapies have to address critical issues for tissue-specific delivery to avoid off-target side effects.

\section{CONCLUSIONS}

As we reviewed above, miRNAs have emerged as critical regulators of cancer metastasis. Accumulating evidence suggests important roles for specific miRNAs in the multi-step process of CRC metastasis. Remarkably, expression levels of miRNAs are associated with clinical relevance in CRC progression and metastasis, indicating they have potential as biomarkers for diagnosis, prognosis prediction, and therapeutic targets. Therefore, future efforts are required to develop clinically applicable miRNA-based tools for predicting prognosis and distant metastasis in CRC patients.

\section{ACKNOWLEDGEMENTS}

This study was supported by the National Research Foundation of Korea (NRF) grant, funded by the Korean government (2014R1A5A2009242).

\section{REFERENCES}

1. Bartel DP (2009) MicroRNAs: target recognition and regulatory functions. Cell 136, 215-233

2. Kim VN, Han J and Siomi MC (2009) Biogenesis of small RNAs in animals. Nat Rev Mol Cell Biol 10, 126-139

3. Friedman JM and Jones PA (2009) MicroRNAs: critical mediators of differentiation, development and disease. Swiss Med Wkly 139, 466-472

4. Siegel R, Desantis C and Jemal A (2014) Colorectal cancer statistics, 2014. CA Cancer J Clin 64, 104-117

5. Kopetz S, Chang GJ, Overman MJ et al (2009) Improved survival in metastatic colorectal cancer is associated with adoption of hepatic resection and improved chemotherapy. J Clin Oncol 27, 3677-3683

6. Rees M, Tekkis PP, Welsh FK, O'Rourke T and John TG (2008) Evaluation of long-term survival after hepatic resection for metastatic colorectal cancer: a multifactorial model of 929 patients. Ann Surg 247, 125-135

7. Nagel R, le Sage C, Diosdado B et al (2008) Regulation of the adenomatous polyposis coli gene by the miR-135 family in colorectal cancer. Cancer Res 68, 5795-5802 
8. Chang TC, Wentzel EA, Kent OA et al (2007) Transactivation of miR-34a by p53 broadly influences gene expression and promotes apoptosis. Mol Cell 26, 745-752

9. Diosdado B, van de Wiel MA, Terhaar Sive Droste JS et al (2009) MiR-17-92 cluster is associated with 13q gain and c-myc expression during colorectal adenoma to adenocarcinoma progression. Br J Cancer 101, 707-714

10. Guo C, Sah JF, Beard L, Willson JK, Markowitz SD and Guda K (2008) The noncoding RNA, miR-126, suppresses the growth of neoplastic cells by targeting phosphatidylinositol 3-kinase signaling and is frequently lost in colon cancers. Genes Chromosomes Cancer 47, 939-946

11. Krichevsky AM and Gabriely G (2009) miR-21: a small multi-faceted RNA. J Cell Mol Med 13, 39-53

12. Akao $Y$, Nakagawa $Y$ and Naoe $T$ (2006) let-7 microRNA functions as a potential growth suppressor in human coIon cancer cells. Biol Pharm Bull 29, 903-906

13. Chen X, Guo X, Zhang H et al (2009) Role of miR-143 targeting KRAS in colorectal tumorigenesis. Oncogene 28, 1385-1392

14. Tsang WP and Kwok TT (2009) The miR-18a* microRNA functions as a potential tumor suppressor by targeting on K-Ras. Carcinogenesis 30, 953-959

15. Hanahan D and Weinberg RA (2000) The hallmarks of cancer. Cell 100, 57-70

16. Friedl $P$ and Wolf K (2003) Tumour-cell invasion and migration: diversity and escape mechanisms. Nat Rev Cancer 3, 362-374

17. Asangani IA, Rasheed SA, Nikolova DA et al (2008) MicroRNA-21 (miR-21) post-transcriptionally downregulates tumor suppressor Pdcd 4 and stimulates invasion, intravasation and metastasis in colorectal cancer. Oncogene 27, 2128-2136

18. Volinia S, Calin GA, Liu CG et al (2006) A microRNA expression signature of human solid tumors defines cancer gene targets. Proc Natl Acad Sci U S A 103, 2257-2261

19. Sayed D, Rane S, Lypowy J et al (2008) MicroRNA-21 targets Sprouty2 and promotes cellular outgrowths. Mol Biol Cell 19, 3272-3282

20. Zhu S, Si ML, Wu H and Mo YY (2007) MicroRNA-21 targets the tumor suppressor gene tropomyosin 1 (TPM1). J Biol Chem 282, 14328-14336

21. Vicinus B, Rubie C, Faust SK et al (2012) miR-21 functionally interacts with the 3'UTR of chemokine CCL20 and down-regulates CCL20 expression in miR-21 transfected colorectal cancer cells. Cancer Lett 316, 105-112

22. Wang $P$, Zou $F$, Zhang $X$ et al (2009) microRNA-21 negatively regulates $\mathrm{Cdc} 25 \mathrm{~A}$ and cell cycle progression in coIon cancer cells. Cancer Res 69, 8157-8165

23. Meng F, Henson R, Wehbe-Janek $H$, Ghoshal K, Jacob ST and Patel T (2007) MicroRNA-21 regulates expression of the PTEN tumor suppressor gene in human hepatocellular cancer. Gastroenterology 133, 647-658

24. Gabriely G, Wurdinger T, Kesari S et al (2008) MicroRNA 21 promotes glioma invasion by targeting matrix metalloproteinase regulators. Mol Cell Biol 28, 5369-5380

25. Slaby O, Svoboda M, Fabian P et al (2007) Altered expression of miR-21, miR-31, miR-143 and miR-145 is related to clinicopathologic features of colorectal cancer. Oncology 72, 397-402
26. Faltejskova P, Besse A, Sevcikova S et al (2012) Clinical correlations of miR-21 expression in colorectal cancer patients and effects of its inhibition on DLD1 colon cancer cells. Int J Colorectal Dis 27, 1401-1408

27. Cottonham CL, Kaneko S and Xu L (2010) miR-21 and miR-31 converge on TIAM1 to regulate migration and invasion of colon carcinoma cells. J Biol Chem 285, 3529335302

28. Chen HY, Lin YM, Chung HC et al (2012) miR-103/107 promote metastasis of colorectal cancer by targeting the metastasis suppressors DAPK and KLF4. Cancer Res 72, 3631-3641

29. Fu J, Tang $W$, Du $P$ et al (2012) Identifying microRNAmRNA regulatory network in colorectal cancer by a combination of expression profile and bioinformatics analysis. BMC Syst Biol 6, 68

30. Huang Z, Huang D, Ni S, Peng Z, Sheng W and Du X (2010) Plasma microRNAs are promising novel biomarkers for early detection of colorectal cancer. Int J Cancer 127, 118-126

31. Kanaan Z, Rai SN, Eichenberger MR et al (2012) Differential microRNA expression tracks neoplastic progression in inflammatory bowel disease-associated colorectal cancer. Hum Mutat 33, 551-560

32. Vickers MM, Bar J, Gorn-Hondermann I et al (2012) Stagedependent differential expression of microRNAs in colorectal cancer: potential role as markers of metastatic disease. Clin Exp Metastasis 29, 123-132

33. Harris TA, Yamakuchi $M$, Ferlito $M$, Mendell JT and Lowenstein CJ (2008) MicroRNA-126 regulates endothelial expression of vascular cell adhesion molecule 1. Proc Natl Acad Sci U S A 105, 1516-1521

34. Lu LF and Liston A (2009) MicroRNA in the immune system, microRNA as an immune system. Immunology 127 , 291-298

35. Bu P, Chen KY, Chen JH et al (2013) A microRNA miR-34a-regulated bimodal switch targets Notch in colon cancer stem cells. Cell Stem Cell 12, 602-615

36. Hwang WL, Jiang JK, Yang SH et al (2014) MicroRNA-146a directs the symmetric division of Snail-dominant colorectal cancer stem cells. Nat Cell Biol 16, 268-280

37. Bitarte N, Bandres E, Boni $V$ et al (2011) MicroRNA-451 is involved in the self-renewal, tumorigenicity, and chemoresistance of colorectal cancer stem cells. Stem Cells 29, 1661-1671

38. Fang Y, Gu X, Li Z, Xiang J and Chen Z (2013) miR-449b inhibits the proliferation of SW1116 colon cancer stem cells through downregulation of CCND1 and E2F3 expression. Oncol Rep 30, 399-406

39. Bonauer A, Carmona G, Iwasaki M et al (2009) MicroRNA-92a controls angiogenesis and functional recovery of ischemic tissues in mice. Science 324, 1710-1713

40. Jain RK (2005) Normalization of tumor vasculature: an emerging concept in antiangiogenic therapy. Science 307, 58-62

41. Poliseno L, Tuccoli A, Mariani L et al (2006) MicroRNAs modulate the angiogenic properties of HUVECs. Blood 108, 3068-3071

42. Xu Q, Liu LZ, Qian X et al (2012) MiR-145 directly targets p70S6K1 in cancer cells to inhibit tumor growth and 
angiogenesis. Nucleic Acids Res 40, 761-774

43. Yamakuchi M, Lotterman CD, Bao C et al (2010) P53-induced microRNA-107 inhibits HIF-1 and tumor angiogenesis. Proc Natl Acad Sci U S A 107, 6334-6339

44. Guo ST, Jiang CC, Wang GP et al (2013) MicroRNA-497 targets insulin-like growth factor 1 receptor and has a tumour suppressive role in human colorectal cancer. Oncogene 32, 1910-1920

45. Sundaram P, Hultine S, Smith LM et al (2011) p53-responsive miR-194 inhibits thrombospondin-1 and promotes angiogenesis in colon cancers. Cancer Res 71, 7490-7501

46. Dews M, Homayouni A, Yu D et al (2006) Augmentation of tumor angiogenesis by a Myc-activated microRNA cluster. Nat Genet 38, 1060-1065

47. Kalluri R and Weinberg RA (2009) The basics of epithelial-mesenchymal transition. J Clin Invest 119, 14201428

48. Heldin $\mathrm{CH}$, Vanlandewijck M and Moustakas A (2012) Regulation of EMT by TGFbeta in cancer. FEBS Lett 586, 1959-1970

49. Thiery JP, Acloque $\mathrm{H}$, Huang RY and Nieto MA (2009) Epithelial-mesenchymal transitions in development and disease. Cell 139, 871-890

50. Burk U, Schubert J, Wellner U et al (2008) A reciprocal repression between ZEB1 and members of the miR-200 family promotes EMT and invasion in cancer cells. EMBO Rep 9, 582-589

51. Gregory PA, Bert AG, Paterson EL et al (2008) The miR-200 family and miR-205 regulate epithelial to mesenchymal transition by targeting ZEB1 and SIP1. Nat Cell Biol 10, 593-601

52. Park SM, Gaur AB, Lengyel E and Peter ME (2008) The miR-200 family determines the epithelial phenotype of cancer cells by targeting the E-cadherin repressors ZEB1 and ZEB2. Genes Dev 22, 894-907

53. Hur K, Toiyama Y, Takahashi M et al (2013) MicroRNA200c modulates epithelial-to-mesenchymal transition (EMT) in human colorectal cancer metastasis. Gut 62, 13151326

54. Gregory PA, Bracken CP, Smith E et al (2011) An autocrine TGF-beta/ZEB/miR-200 signaling network regulates establishment and maintenance of epithelial-mesenchymal transition. Mol Biol Cell 22, 1686-1698

55. Rokavec M, Oner MG, Li H et al (2014) IL-6R/STAT3/ miR-34a feedback loop promotes EMT-mediated colorectal cancer invasion and metastasis. J Clin Invest 124, 1853-1867

56. Booth RA (2007) Minimally invasive biomarkers for detection and staging of colorectal cancer. Cancer Lett 249,
87-96

57. Habermann JK, Bader FG, Franke C et al (2008) From the genome to the proteome--biomarkers in colorectal cancer. Langenbecks Arch Surg 393, 93-104

58. Soreide K, Nedrebo BS, Knapp JC, Glomsaker TB, Soreide JA and Korner H (2009) Evolving molecular classification by genomic and proteomic biomarkers in colorectal cancer: potential implications for the surgical oncologist. Surg Oncol 18, 31-50

59. Duffy MJ (2001) Carcinoembryonic antigen as a marker for colorectal cancer: is it clinically useful? Clin Chem 47, 624-630

60. Mandel JS, Bond JH, Church TR et al (1993) Reducing mortality from colorectal cancer by screening for fecal occult blood. Minnesota Colon Cancer Control Study. N Engl J Med 328, 1365-1371

61. Kanaan Z, Roberts H, Eichenberger MR et al (2013) A plasma microRNA panel for detection of colorectal adenomas: a step toward more precise screening for colorectal cancer. Ann Surg 258, 400-408

62. Wang S, Xiang J, Li Z et al (2015) A plasma microRNA panel for early detection of colorectal cancer. Int J Cancer 136, 152-161

63. Toiyama Y, Takahashi M, Hur K et al (2013) Serum miR-21 as a diagnostic and prognostic biomarker in colorectal cancer. J Natl Cancer Inst 105, 849-859

64. Toiyama Y, Hur K, Tanaka K et al (2014) Serum miR200c is a novel prognostic and metastasis-predictive biomarker in patients with colorectal cancer. Ann Surg 259, 735-743

65. Hur K, Toiyama Y, Schetter AJ et al (2015) Identification of a Metastasis-Specific MicroRNA Signature in Human Colorectal Cancer. J Natl Cancer Inst 107

66. Wang CJ, Stratmann J, Zhou ZG and Sun XF (2010) Suppression of microRNA-31 increases sensitivity to 5-FU at an early stage, and affects cell migration and invasion in HCT-116 colon cancer cells. BMC Cancer 10, 616

67. Akao Y, Noguchi S, lio A, Kojima K, Takagi T and Naoe T (2011) Dysregulation of microRNA-34a expression causes drug-resistance to 5-FU in human colon cancer DLD-1 cells. Cancer Lett 300, 197-204

68. Nakagawa $Y$, linuma $M$, Naoe $T$, Nozawa $Y$ and Akao $Y$ (2007) Characterized mechanism of alpha-mangostin-induced cell death: caspase-independent apoptosis with release of endonuclease-G from mitochondria and increased miR-143 expression in human colorectal cancer DLD-1 cells. Bioorg Med Chem 15, 5620-5628

69. Ng EK, Tsang WP, Ng SS et al (2009) MicroRNA-143 targets DNA methyltransferases $3 \mathrm{~A}$ in colorectal cancer. $\mathrm{Br}$ Cancer 101, 699-706 